\title{
Deformable Sphere Impact on Resting Droplets
}

\author{
Rafsan Rabbi ${ }^{1}$, Akihito Kiyama ${ }^{1}$, John S. Allen ${ }^{2} \&$ Tadd Truscott $^{1,3^{*}}$ \\ ${ }^{1}$ Utah State University, ${ }^{2}$ University of Hawaii at Manoa, ${ }^{3}$ King Abdullah University of Science and Technology \\ *Corresponding author: tadd.truscott@kaust.edu.sa
}

Several different rebound behaviors occur when a solid object impacts a resting liquid droplet. High-speed imaging reveals the various droplet rebound phenomena from two spheres with very different elasticities.

Two similarly sized spheres of $16 \mathrm{~mm}$ diameter and density $\left(\approx 1100 \mathrm{~kg} / \mathrm{m}^{3}\right)$ were dropped from $75 \mathrm{~mm}$ onto the center of a water droplet resting on a superhydrophobic substrate with a static contact angle of $\approx 141^{\circ}$ (Figure 1 ). The difference between the spheres is that one is a deformable water bead with a shear modulus of $\mathrm{G} \approx 0.007 \mathrm{MPa}$ and the other is a polystyrene solid sphere of $\mathrm{G} \approx 750 \mathrm{MPa}$. The droplet dynamics were recorded with a Phantom v2511 high-speed color camera at 20,000 fps. To enhance the visual contrast of the solid sphere (orange), we dyed water beads with blue food coloring and employed frontillumination. A more detailed experimental method can be found in [1].

The water droplet reveals one of three distinguishable behaviors. If the sphere is deformable, droplets can show noticeable spreading after the impact (figure $2 a \& b)$, whereas the spreading is suppressed when the sphere is less deformable, and the water creates a bridge between the substrate for the non-deformable sphere (figure 2c). In other words, if the droplet spreading speed is large compared to the elastic wave speed within the sphere the droplet will spread easily. When the impact speed is sufficient, the droplet spreads quickly before it detaches from the substrate (figure $2 b$ ).

The images presented in this work were first presented at [2], and the detailed scientific content is reported in [1].

[1] Rabbi, R., Kiyama, A., Allen, J., and Truscott, T., 2021, "Droplet Lift-off from Hydrophobic Surface from Impact with Soft Hydrogel Spheres", Preprint, In revision at Nat. Commun. https: //doi:10.21203/rs.3.rs-859493/v1.

[2] Rabbi, R., Allen, J., and Truscott, T., 2019, "Viscoelasticity Gives You Wings!", 2019 APS/DFD Gallery of Fluid Motion Video entry, V0062. https://doi.org/10.1103/APS.DFD.2019.GFM.V0062.

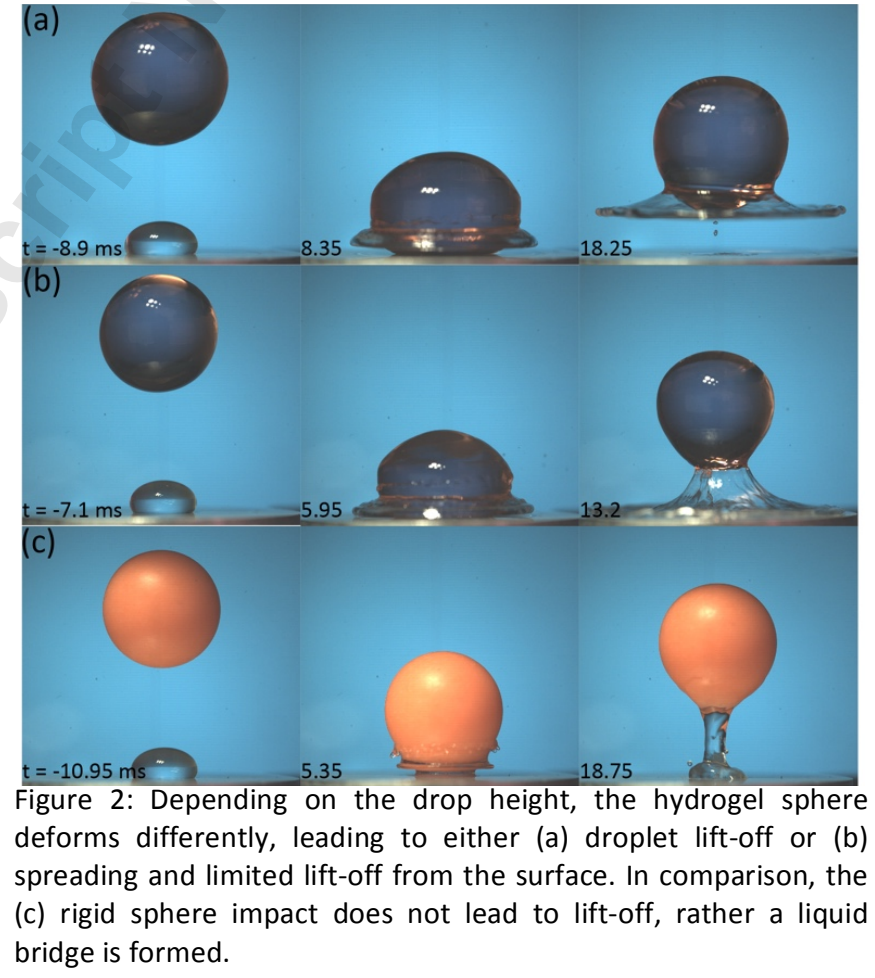

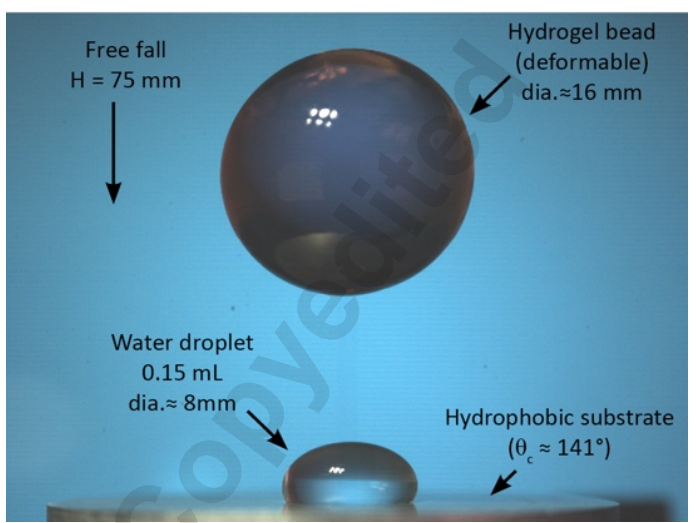

Figure 1: Deformable hydrogel sphere impacting a resting water droplet. 